\title{
Spectrum of imaging findings in pulmonary infections. Part 2: Fungal, mycobacterial, and parasitic
}

\author{
Mandeep Garg A,B,D,E,F, Nidhi Prabhakar ${ }^{A, B, E, F}$, Ajay Gulati D,E,F, Ritesh Agarwal ${ }^{B, E, F}$, Sahajal Dhooria ${ }^{B, E, F}$ \\ Postgraduate Institute of Medical Education and Research, India
}

\begin{abstract}
Chest radiography is generally the first imaging modality used for the evaluation of pneumonia. It can establish the presence of pneumonia, determine its extent and location, and assess the response to treatment. Computed tomography is not used for the initial evaluation of pneumonia, but it may be used when the response to treatment is unusually slow. It helps to identify complications, detect any underlying chronic pulmonary disease, and characterise complex pneumonias. Although not diagnostic, certain imaging findings may suggest a particular microbial cause over others. Knowledge of whether pneumonia is community-acquired or nosocomial, as well as the age and immune status of the patient, can help to narrow the differential diagnoses. The purpose of this article is to briefly review the various pulmonary imaging manifestations of pathogenic organisms. This knowledge, along with the clinical history and laboratory investigations of the patient, may help to guide the treatment of pneumonia.
\end{abstract}

Key words: fungal, bacterial, pneumonia, viral, mycobacterial, parasitic.

\section{Fungal pneumonia}

Fungi causing pneumonia can be of two types: either pathogenic, which can infect anyone (coccidiomycosis, blastomycosis, histoplasmosis), or saprophytic, which infects immunocompromised people (pneumocystis, candidosis, aspergillosis, mucormycosis) [1].

Aspergillus fumigatus is ubiquitous. It infects immunocompromised or susceptible individuals. Its pulmonary manifestations are of five types: simple aspergilloma, allergic bronchopulmonary aspergillosis (ABPA), airway invasive pulmonary aspergillosis, angioinvasive pulmonary aspergillosis, and chronic pulmonary aspergillosis (Figure 1).

Aspergilloma is a ball of coalescent mycelial hyphae. It typically colonises pre-existing cavities, which may be formed due to a predisposing condition like tuberculosis, sarcoidosis, etc. On chest radiograph, they are seen as round/oval lesions lying within a cavity. Sometimes an 'air crescent' sign is seen, in which curvilinear airspace is seen separating the fungal ball from the cavity. Com- puted tomography (CT) shows a similar finding in which the fungal ball is seen as a sponge-like mass with air spaces, which moves with the change in the position of the patient [2].

ABPA is associated with asthma, elevated IgE, positive skin reactivity to Aspergillus, and positive serum precipitins. This is caused by a complex hypersensitivity reaction to fungus, leading to the proliferation of fungi in the airway lumen, and leading in turn to bronchial dilatation and mucus impaction. Chest radiograph shows central bronchiectasis and mucus-filled impacted bronchi (seen as branching opacities - 'gloved-finger sign'). CT shows bronchiectasis and mucus-impacted bronchi, predominant in the upper lobes. Mucus in bronchi may show high density, probably due to calcium deposits [3].

Airway invasive aspergillosis shows Aspergillus present deep to the airway basement membrane on histological analysis. It is seen in immunocompromised patients. It can manifest as acute tracheobronchitis, bronchiolitis, or bronchopneumonia. Tracheobronchitis will be seen as tracheal and/or bronchial wall thickening on imaging, or imaging

Correspondence address:

Dr. Mandeep Garg, MD, FRCR, Prof., Department of Radiodiagnosis and Imaging, PGIMER, Chandigarh, India, e-mail: gargmandeep@hotmail.com

Authors' contribution:

A Study design · B Data collection · C Statistical analysis · D Data interpretation · E Manuscript preparation · F Literature search · G Funds collection 

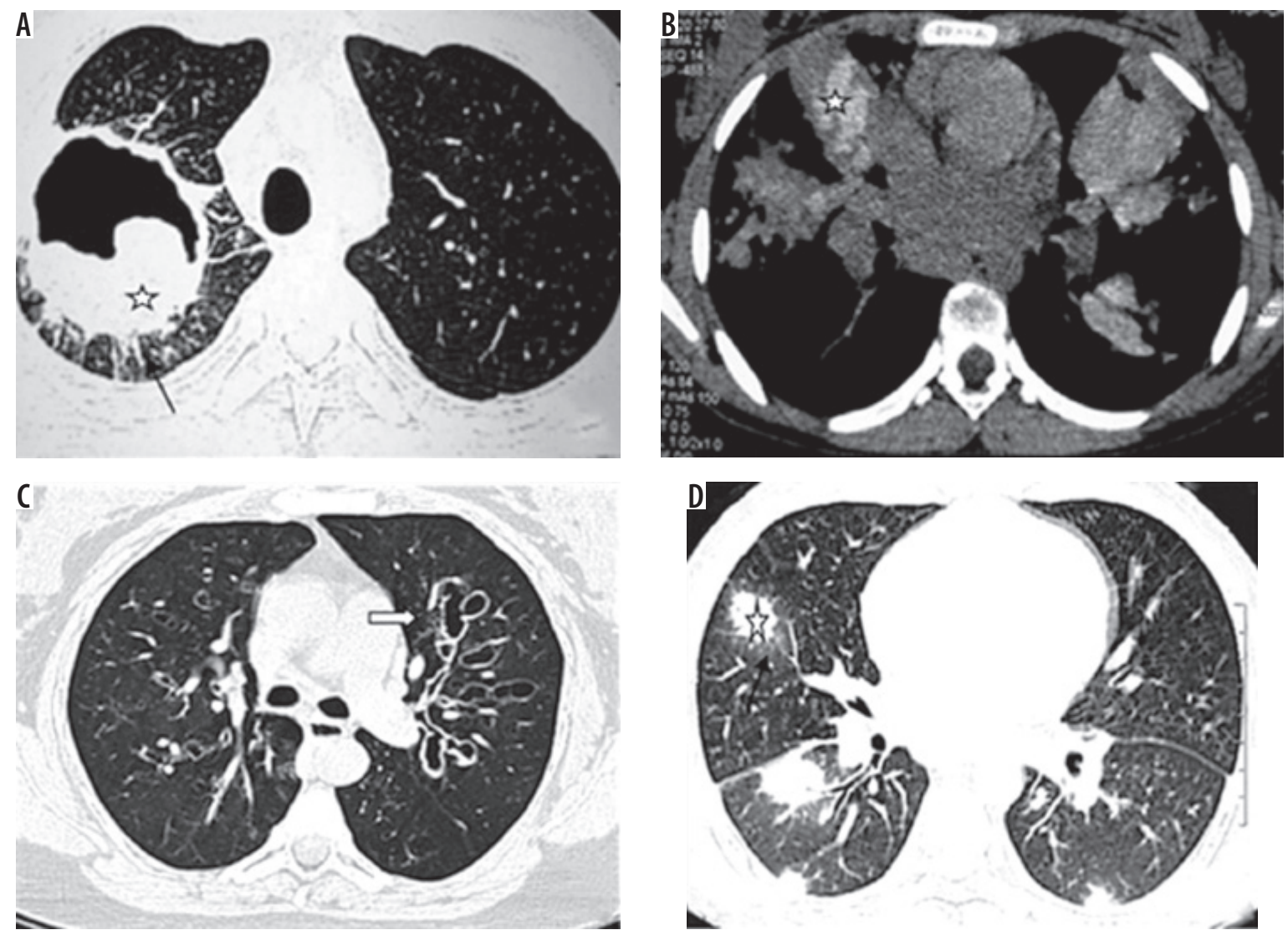

Figure 1. Spectrum of findings in Aspergillus infection. A) Chest computed tomography (CT) shows the presence of fibro-cavitary lesion (black arrow) with aspergilloma (asterisk), in a patient treated for pulmonary tuberculosis. B, C) Chest (T in a patient of asthma shows high attenuating mucus (asterisk in B) and central bronchiectatic changes (white block arrow in C) suggestive of allergic bronchopulmonary aspergillosis. D) Angioinvasive aspergillosis in a post renal transplant patient. Chest CT showing the presence of random nodules (asterisk) with adjacent ground glass opacities (black arrow)

findings may be normal. CT findings in bronchiolitis include centrilobular nodules and branching or linear opacities with 'tree in bud' appearance. Bronchopneumonia is seen as peribronchial areas of consolidation [3].

Angioinvasive aspergillosis is seen exclusively in the immunocompromised host, predominantly in patients with neutropaenia. This pulmonary infection is due to invasion of small to medium arteries leading to the development of necrotic haemorrhagic nodules or infarcts in lungs. Imaging findings include nodules or wedgeshaped opacities, frequently in subpleural areas. Nodules may show ground glass opacity (GGO) around them due to haemorrhage (CT halo sign). Cavitation or air crescent sign may be seen in the period of convalescence, approximately 2-3 weeks after treatment. CT pulmonary angiography may show 'vessel occlusion sign' [4].

Chronic pulmonary aspergillosis infection is the less severe and more localised form of aspergillosis infection. It is seen in patients with mild immunosuppression, e.g. in patients of diabetes, malnutrition or, with pre-existing lung disease. It is of three types: chronic cavitating, chronic necrotising, and chronic fibrosing aspergillosis. Chronic cavitating shows cavities with or without fungal balls in upper lobes. Chronic necrotising is relatively more aggressive and hence is also called semi-invasive aspergillosis. There is the presence of cavities and areas of consolidation with breakdown on CT, and the air-crescent sign may also be seen. Chronic fibrosing type shows fibrocavitary lesions, parenchymal, and pleural fibrosis. All these chronic infections are difficult to differentiate from tuberculosis, actinomycosis, and histoplasmosis on imaging [2].

Cryptococcus neoformans is a yeast form of fungus, which is found in soil or bird droppings. It is more common in immunocompromised patients. The chest radiograph may show subpleural single or multiple nodules ranging in size from 0.5 to $4 \mathrm{~cm}$. Nonspecific perihilar, diffuse nodular or reticulonodular patterns may be seen in acquired immunodeficiency syndrome (AIDS) patients. CT shows single or multiple pulmonary nodules or areas of segmental/lobar consolidation (Figure 2) [5]. Cavitation within nodules and large areas of consolidation are more common in immunocompromised patients [6]. Miliary pattern of nodules or pleural effusions may be seen. Hilar or mediastinal lymph nodes may be seen. In immunocompetent patients, it usually heals spontaneously [7].

Histoplasma is found in soil and bird excreta. Usually, subclinical infection occurs, which leads to the formation of calcified nodules [8]. Imaging findings resemble tuberculosis, showing areas of consolidation with necrosis/cavitation and nodules. Hilar and mediastinal enlarged lymph nodes may be seen. The chronic form resembles post-primary tuberculosis showing fibrosis, volume loss, and calcifica- 

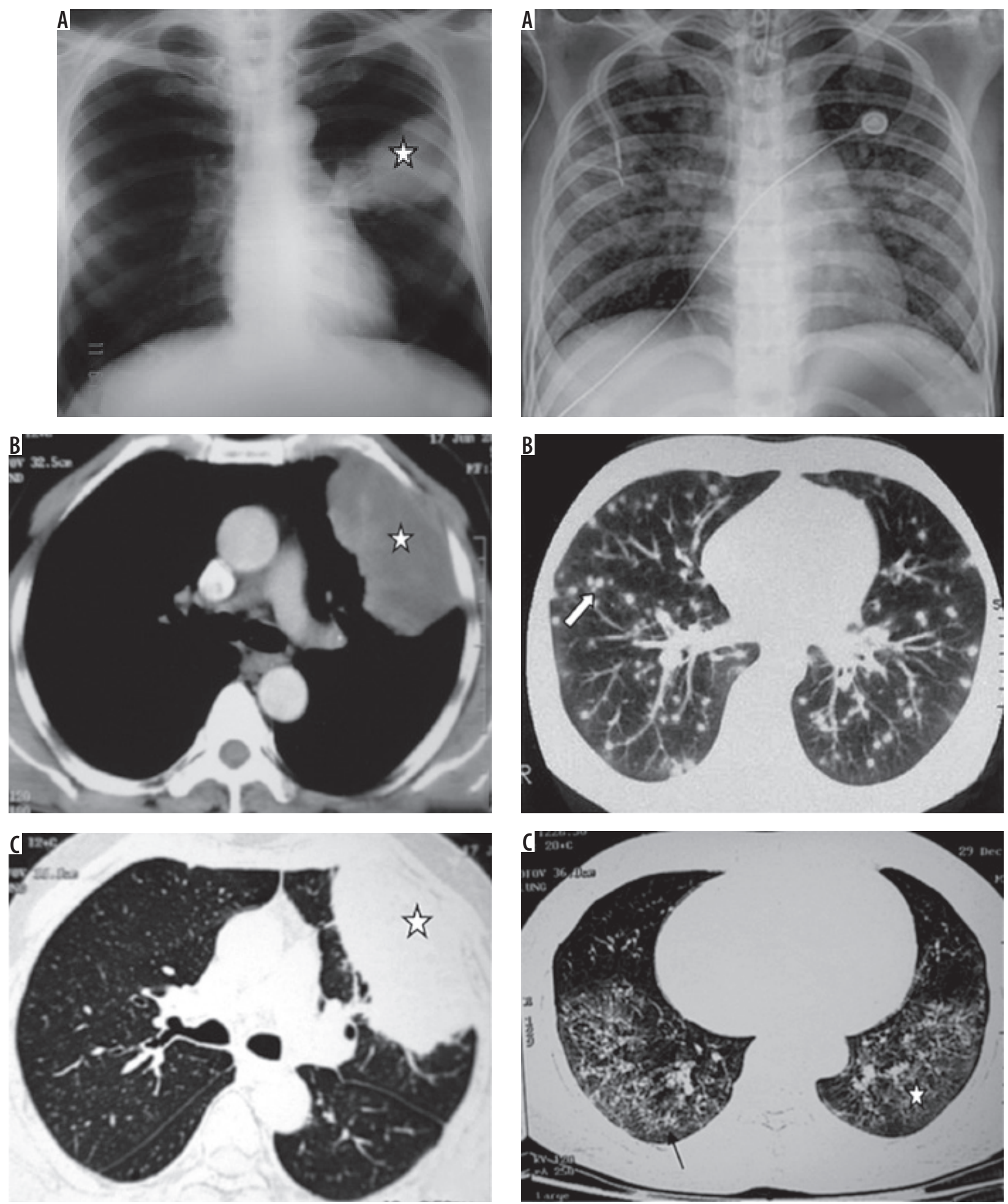

Figure 2. Post renal transplant patient with cryptococcoma (proven by partial lobectomy). A) Chest radiograph shows mass-like consolidation in left mid lung zone (asterisk). B, C) Chest computed tomography image shows peripheral wedge-shaped hypodense mass like consolidation in left upper lobe (asterisk)

tion. Another type may be seen in immunocompromised or young patients, in whom small miliary nodules are seen diffusely scattered in both lungs (Figure 3) [9].

Pneumocystis jiroveci is seen in AIDS patients, organ transplant recipients, or patients undergoing chemothe-

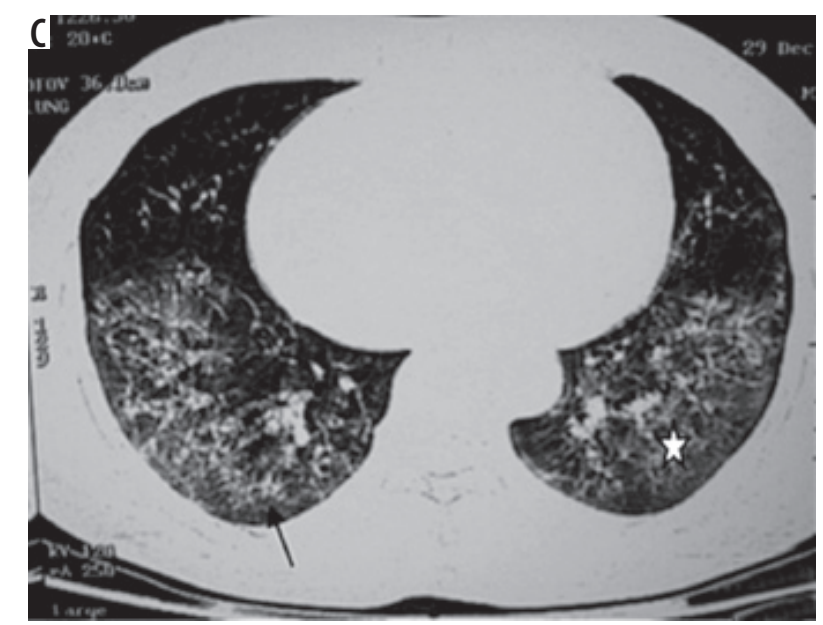

Figure 3. Patient with disseminated histoplasmosis. A) Chest radiograph shows presence of nodules in both lungs. B) Chest computed tomography (CT) shows small random nodules (arrow) in both lungs. C) Disseminated histoplasmosis in a different patient. Chest CT shows small nodules with intralobular septal thickening (black arrow) and ground glass opacities (asterisk) in both lungs

rapy or on steroids. Pneumocystis has become less frequent due to antibiotic prophylaxis and retroviral therapy in AIDS patient. The chest radiograph may be normal or may show perihilar opacities or diffuse infiltrates. CT shows perihilar GGO or consolidation in patchy or geo- 

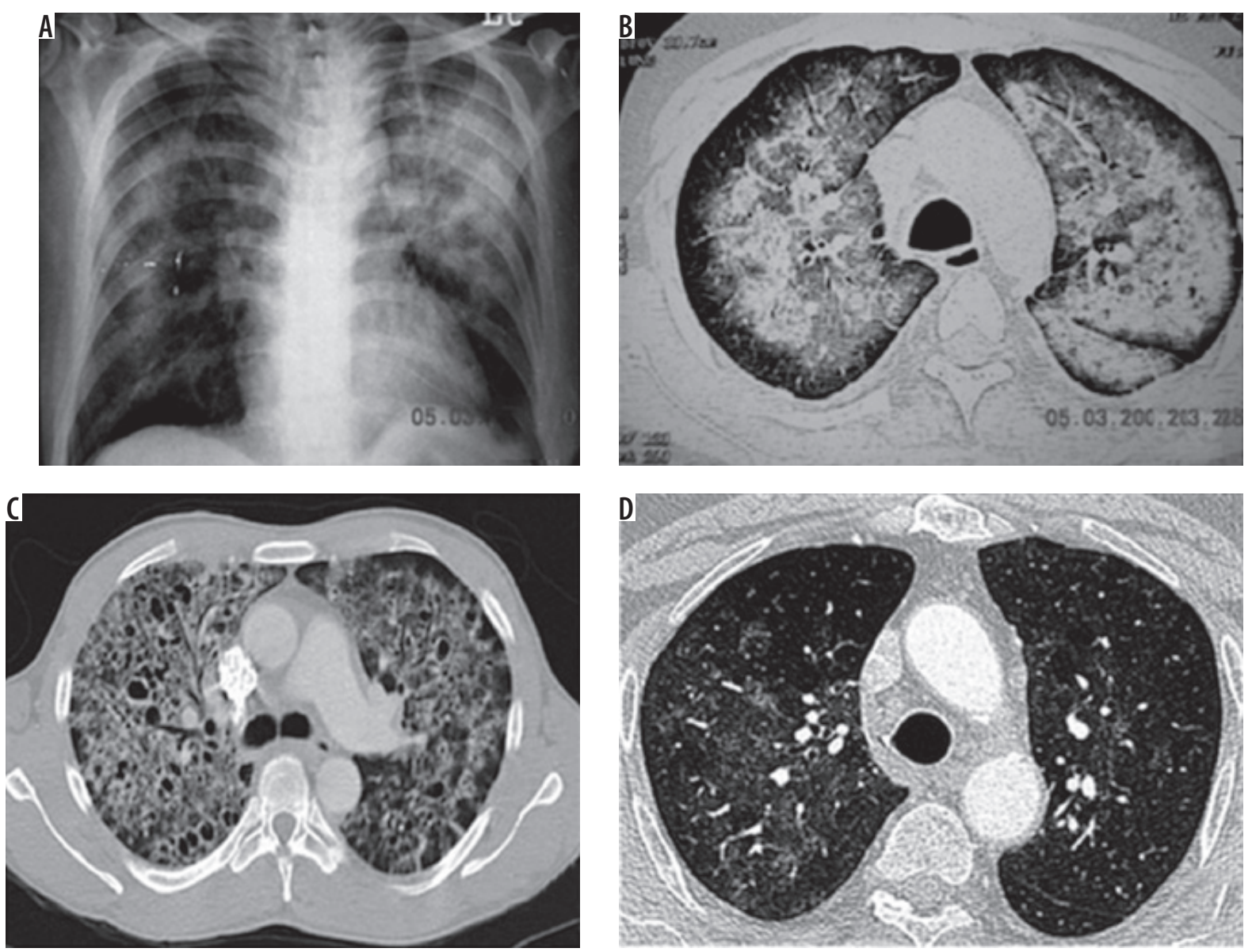

Figure 4. Retropositive patient with Pneumocystis jiroveci pneumonia. A) Chest radiograph shows reticulonodular opacities and areas of consolidation in both lungs. B) Chest computed tomography (CT) image shows presence of ground glass opacities with intralobular septal thickening. C) Chest (T in a different patient of Pneumocystis jirovecii shows ground glass opacities with multiple cysts and septal thickening. D) Chest (T in another patient with Pneumocystis jirovecii pneumonia shows patchy areas of ground glass opacities
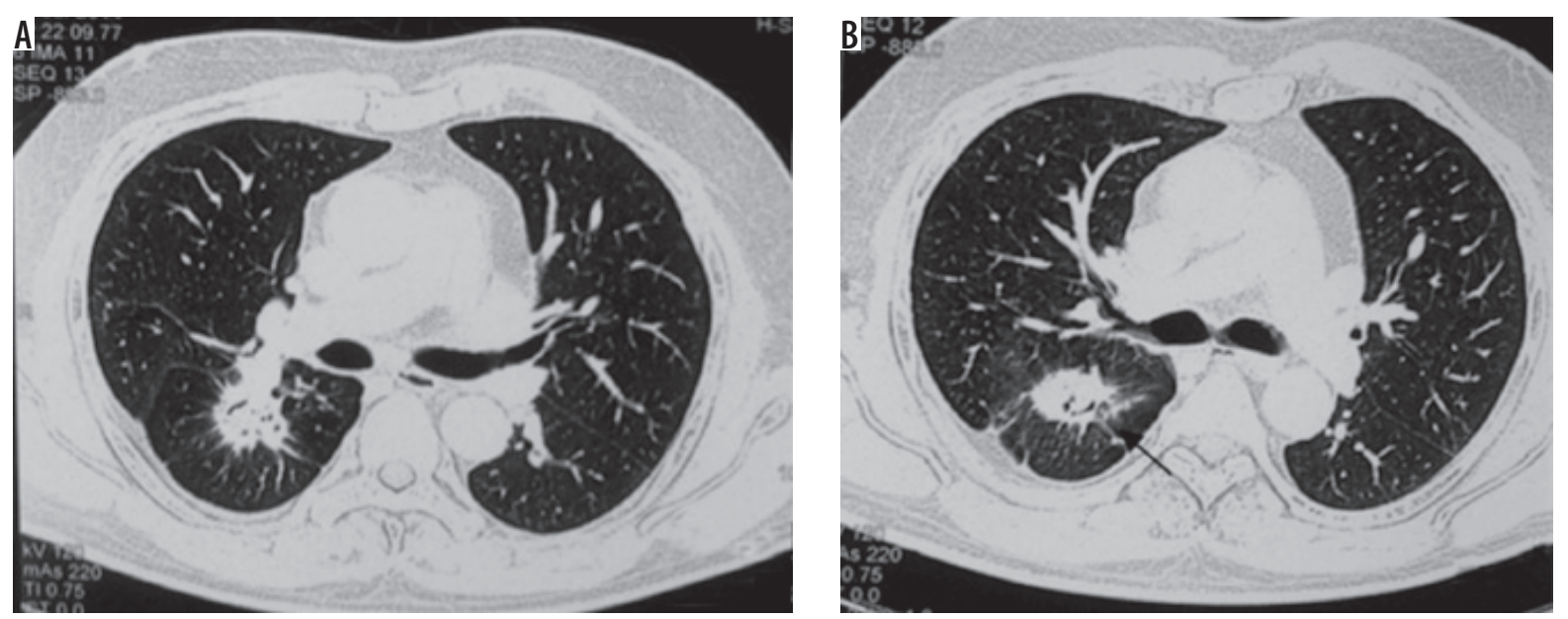

Figure 5. Patient with uncontrolled type 2 diabetes mellitus, fine needle aspiration-proven mucormycosis. A, B) Chest computed tomography images show presence of nodular areas of consolidation with breakdown in right lower lobe with adjacent ground glass opacities (black arrow in B)

graphic distribution [10]. Thin-walled cavities may develop (Figure 4). Pneumothorax may be seen as a complication. Less commonly, miliary disease, pulmonary nodules, pleural effusion, and mediastinal lymphadenopathy may develop.

Mucormycosis infection is caused by inhalation of spores. Paranasal sinuses and lungs are most commonly affected. Risk factors include diabetes mellitus, immuno- suppression, haematological malignancy, and stem cell or solid organ transplant recipients [11]. Imaging findings are non-specific [12]. Chest radiograph shows lobar/multilobar consolidation, solitary or multiple nodules and masses. Cavitation is seen in $40 \%$ of cases. CT shows nodules or consolidation with GGO. Areas of consolidation show cavitation on subsequent imaging (Figure 5) [13]. The reversed halo 


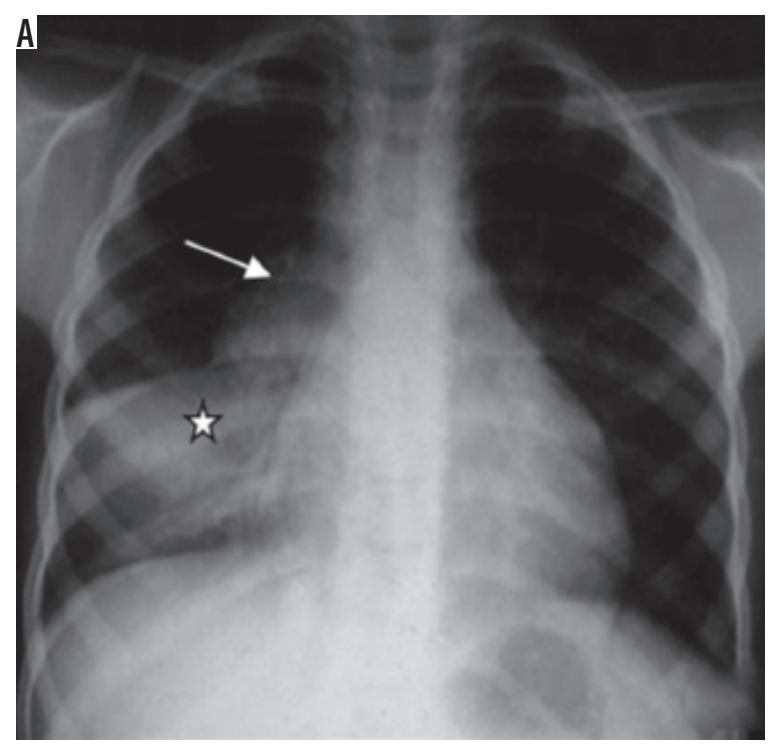

sign, i.e the focal area of GGO within the surrounding peripheral rim of consolidation, can also be seen on CT [13]. Lymphadenopathy and pleural effusion may be seen.

\section{Mycobacterial pneumonia}

Mycobacterial tuberculosis accounts for 95\% of pulmonary mycobacterial infections. Predisposing factors include HIV, poverty, malnutrition, diabetes, silicosis, and malignancy. According to the classic teaching, pulmonary tuberculosis is mainly of two types: primary tuberculosis, which is an initial infection in an unsensitised individual, and post-primary tuberculosis, which is primarily due to reactivation of latent foci of infection implanted during primary infection or, rarely, due to reinfection by tubercular bacteria [14].

Primary tuberculosis is seen in infants and children. It may also be seen in adults in developed countries [15]. Organisms usually reach alveoli in subpleural areas and multiply, leading to regions of peripheral consolidation with the involvement of lymphatics and enlargement of regional lymph nodes (Figure 6) [16]. Pleural effusion may be seen in up to $38 \%$ of cases [17]. The most common radiographic appearance of primary tuberculosis is a normal radiograph [16]. In one third of patients, ill-defined rounded or linear opacity remains, with or without calcification; this is called Ghon's focus. Ghon's focus along with calcified lymph nodes is called Ranke complex. However, if the immune response is weak, the primary infection develops into consolidation, cavitation, and bronchogenic spread.

Post-primary tuberculosis is usually seen in adults [18]. The abnormality is generally seen in apical and posterior segments of upper lobes or superior segments of lower lobes. An ill-defined area of consolidation is the earliest finding. Cavitation usually occurs in areas of consolidation. Walls of cavities may be thin to thick or smooth to nodular [19]. Endobronchial spread leads to a typical treein-bud appearance (Figure 7). Pleural effusion is seen in

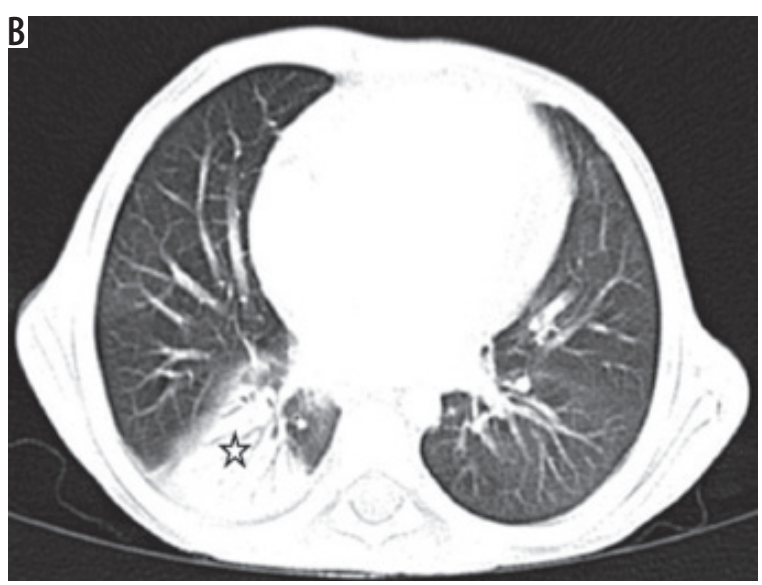

Figure 6. Patient with sputum positive pulmonary tuberculosis. A) Chest radiograph shows bulky right hilum (white arrow) consistent with hilar lymphadenopathy with lower lobar consolidation (asterisk). B) Chest computed tomography shows consolidation in right lower lobe (asterisk)

up to $18 \%$ of cases of post-primary tuberculosis [19]. Pyopneumothorax/pneumothorax may develop. Lymphadenopathy is uncommon in post-primary tuberculosis. In the chronic stage, fibrosis is seen in lung parenchyma, which may lead to volume loss and bronchiectasis. Calcification and pleural thickening may be seen. Chronic fibrous cavities may be prone to secondary infection or fungal colonisation.

The use of newer molecular epidemiological techniques disapproves of the theory that the radiographic appearance of tuberculosis depends on the time since infection. Upper lobe cavitary disease, which is more commonly seen in adults, is the disease of immunocompetent patients, while the lower zone disease with lymphadenopathy and pleural effusions which is more commonly seen in children, is the disease of immunocompromised patients [20].

Tracheobronchial tuberculosis is seen in approximately $10-39 \%$ of cases of pulmonary tuberculosis [21], usually due to the rupture of lymph nodes into a bronchus. Rasmussen aneurysm is formed due to the granulomatous weakening of the pulmonary artery wall; it is a life-threatening complication. In some patients, a miliary pattern is seen, in which multiple tiny random nodules (1-2 mm) are scattered in both lungs (Figure 7D) [22]. In 5\% of patients of reactivation tuberculosis, tuberculoma may be seen, which is a sharply marginated round or oval lesion (0.4-5 cm in size) [23]. Sometimes, it can mimic malignancy. Its central part contains caseous material, and the peripheral portion contains epithelioid histiocytes, multinucleated giant cells, and collagen. Chest wall involvement in tuberculosis may be due to direct spread from lung or haematogeneous seeding. It may affect soft tissue, ribs, or cartilage. Fibrosing mediastinitis is an uncommon complication, in which CT shows a mediastinal or hilar mass, which may sometimes show calcification. It can cause tracheobronchial or pulmonary vessel narrowing and sometimes may lead to SVC syndrome. 

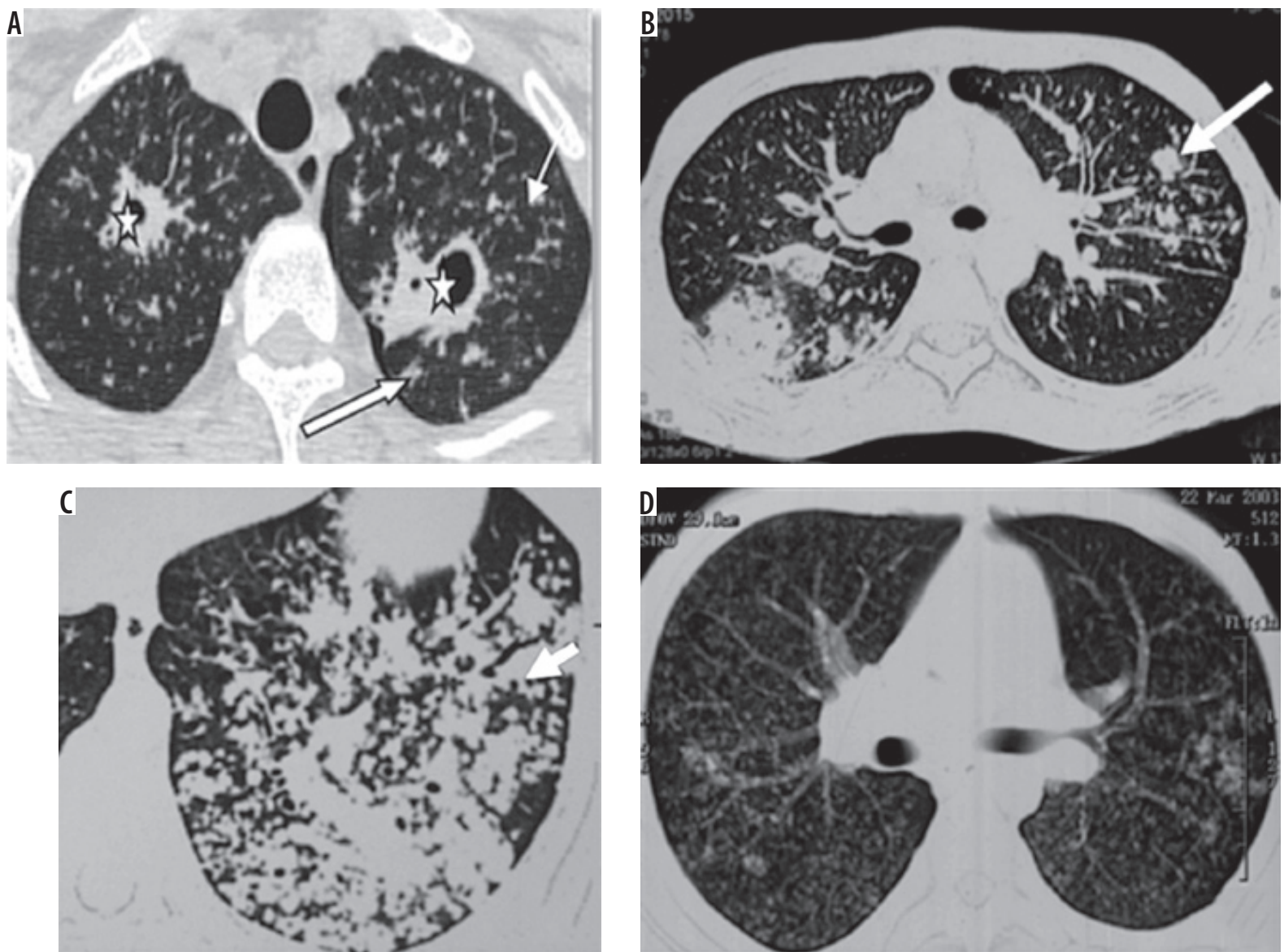

Figure 7. Patient with post primary tuberculosis. A) Chest computed tomography (CT) image shows cavitatory lesions (asterisk) with multiple centrilobular nodules (white arrows) in apical segment of right upper lobe and apicoposterior segment of left upper lobe. B, C) Multiple coalescing peribronchial nodules in left lung (long white block arrow in B, white short block arrow in C). D) Chest (T image shows few centrilobular and multiple tiny random nodules (consistent with miliary tuberculosis)

The common non-tuberculosis mycobacteria that cause infections are Mycobacterium avium-intracellulare or M. kansasii [24]. CT findings of non-tubercular mycobacterial infection include bronchiectasis, small and large nodules, consolidation, centrilobular nodules, and cavitary lesions [25]. Non-tubercular mycobacterial infection shows less surrounding parenchymal infiltrate, less bronchogenic spread and more contiguous spread, and more involvement of pleura, as compared to tubercular mycobacterial infection. Cavities in non-tubercular mycobacterial infection are thinner and with even walls. In addition, ill-defined satellite tree-in-bud nodules and non-cavitary nodules $>10 \mathrm{~mm}$ are more suggestive of non-tubercular mycobacterial infection [26]. Non-tuberculosis mycobacterial infections involve middle and lower lobes and bilateral lungs more frequently (Figure 8) [27].

\section{Parasitic pneumonia}

Parasite infections occur in both immunocompromised and immunocompetent patients. They occur worldwide, and with increasing travel and immigration, they are growing in prevalence. It is important to identify them early because most of them are curable with medical or surgical treatment.
Echinococcus granulosus and E. multilocularis are the most common organisms responsible for the formation of pulmonary hydatid cysts. The reservoir is dogs. Intermediate hosts are mammals. Humans are accidental hosts and ova are acquired from fomites, contaminated in water or directly from sheep, dogs, or cats. Larvae develop in the duodenum, enter the blood, and reach other organs. Lungs are the second most common organ of involvement in adults, after the liver. Lungs are the most common site of involvement in children [28]. $10 \%$ are associated with hepatic hydatid cysts [1]. Hydatid cysts consist of three layers, pericyst, ectocyst, and endocyst. They can be ruptured or unruptured at presentation. Unruptured hydatids on CT are seen as well demarcated spherical or oval homogeneous cystic lesions with enhancing walls in the lung, more commonly in mid and lower zones. Daughter cysts may be seen as curved septations within it. Calcification is rare in pulmonary hydatid cysts. The following radiographic features may be seen: a 'meniscus sign or crescent sign' is formed when there is erosion of adjacent bronchioles by hydatid cyst, leading to air between the layers of hydatid cyst; an 'onion sign' is seen when there is meniscus sign and also air-fluid level within endocyst [29]; a 'water lily sign' is seen due to floating membranes on fluid in cyst; a 'dry cyst sign' is seen when there are no contents in cyst due to expectora- 

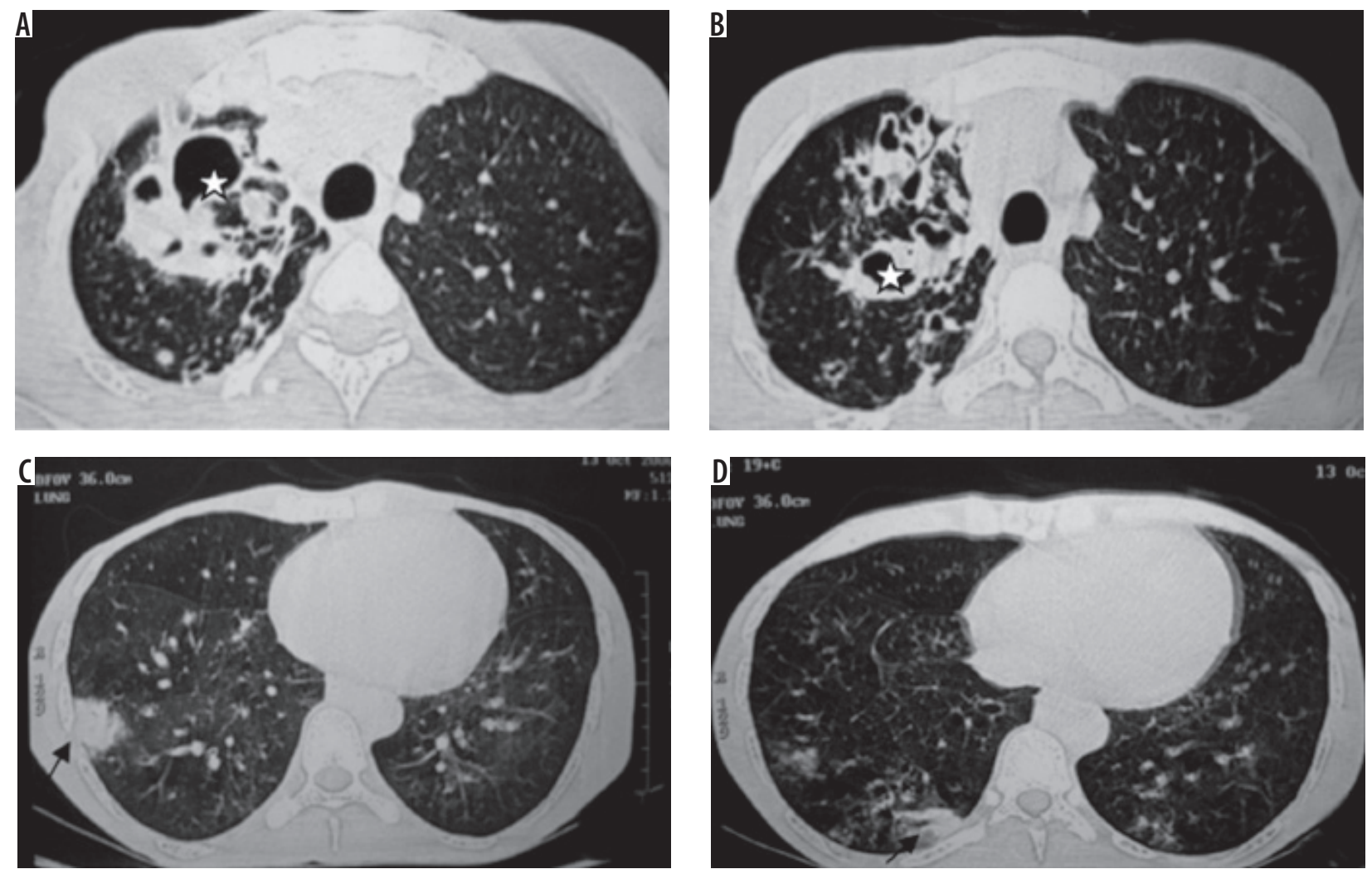

Figure 8. Retropositive patient with Mycobacterium avium intracellulare complex infection. Chest computed tomography images show presence of bronchiectatic changes (asterisk in A and B) in right upper lobe with associated ground glass opacities and consolidation in right lower lobe (black arrows in C and D)

tion of contents; and a 'serpent sign' is seen when there are membranes in the dependent part of a dry cyst (Figure 9) [30]. Hydatid cysts may rupture into pleural space, which may lead to effusion or hydropneumothorax. Cyst rupture can also lead to secondary infection or lung abscess formation.

Pleuropulmonary involvement is the most frequent manifestation of extraintestinal amoebiasis, after liver abscess [31]. A liver abscess can directly extend and involve pleura and lung (occurs in 6-40\% of hepatic abscess patients) [31]. Radiological findings include elevated right hemidiaphragm, pleural effusion/empyema, lower lobe abscess or consolidation, and hepatomegaly (Figure 10). Haziness and loss of definition of the right hemidiaphragm are the earliest radiographic findings. If there is communication of lung abscess to bronchi, the patient may present with 'anchovy paste'-like sputum. USG can be helpful in revealing liver abscess and pleural collection.

Ascaris lumbricoides migrate from the small intestine to pulmonary vessels, where they mature and cause capillary wall destruction, followed by oedema and haemorrhage. Patient clinically presents with fever, cough, and blood and sputum eosinophilia. Larvae may be seen in sputum. Radiographically, unilateral or bilateral migratory pulmonary non-segmental consolidations are observed, which typically clear within 10 days. They may coalesce and increase in severe cases. Löffler's syndrome is associated with ascariasis, in which patients typically show transient radiographic abnormality, minimal con- stitutional symptoms, and peripheral eosinophilia (Figure 11) [32].

\section{Conclusions}

Pneumonia is an important cause of morbidity and mortality in the general population. Imaging plays an essential role in the diagnosis and treatment of pneumonia. Chest radiography should be the first investigation used whenever pneumonia is suspected [33]. CT is used when the findings are inconclusive on chest radiograph or when there is a suspicion of a complication or an underlying chronic lung disease. The imaging findings of various pathogenic organisms overlap, and it is not possible to determine the aetiological agent on imaging alone. However, the radiological findings along with a knowledge of whether pneumonia is community-acquired or nosocomial and the age and the immune status of the patient, can help to narrow the differential diagnosis of the causative organism. It is important to know the various imaging manifestations of pathogenic organisms, because this knowledge along with the clinical history and laboratory investigations of the patient can be helpful in arriving at a diagnosis and guiding the management of the patient.

\section{Conflict of interest}

The authors report no conflict of interest. 

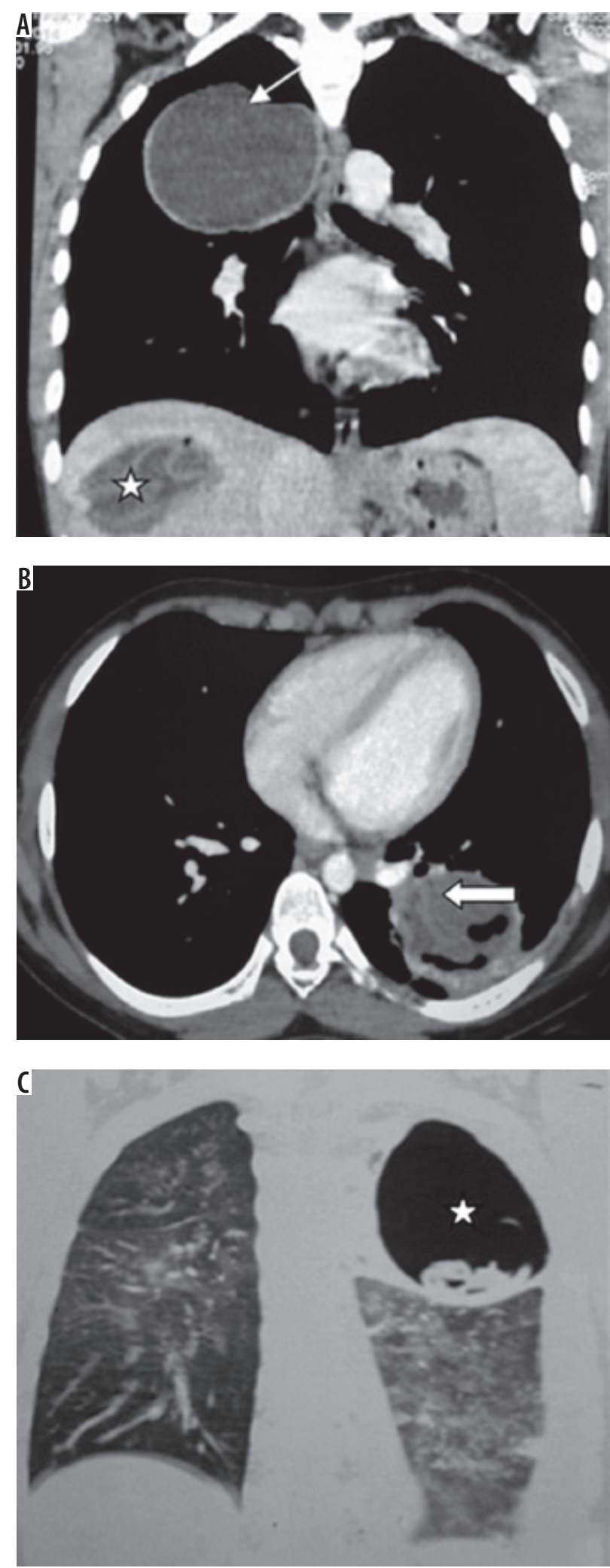

Figure 9. A) Chest computed tomography (CT), coronal reformatted image shows presence of unilocular cystic lesion in right upper lobe (white arrow). Note the presence of cyst with collapsed membrane in visualised right lobe of liver (asterisk). B) Collapsed membranes within the cyst in left lower lobe (white block arrow). C) Chest CT, coronal reformatted image, shows presence of thin-walled cavitary lesion with collapsed membranes within (asterisk)
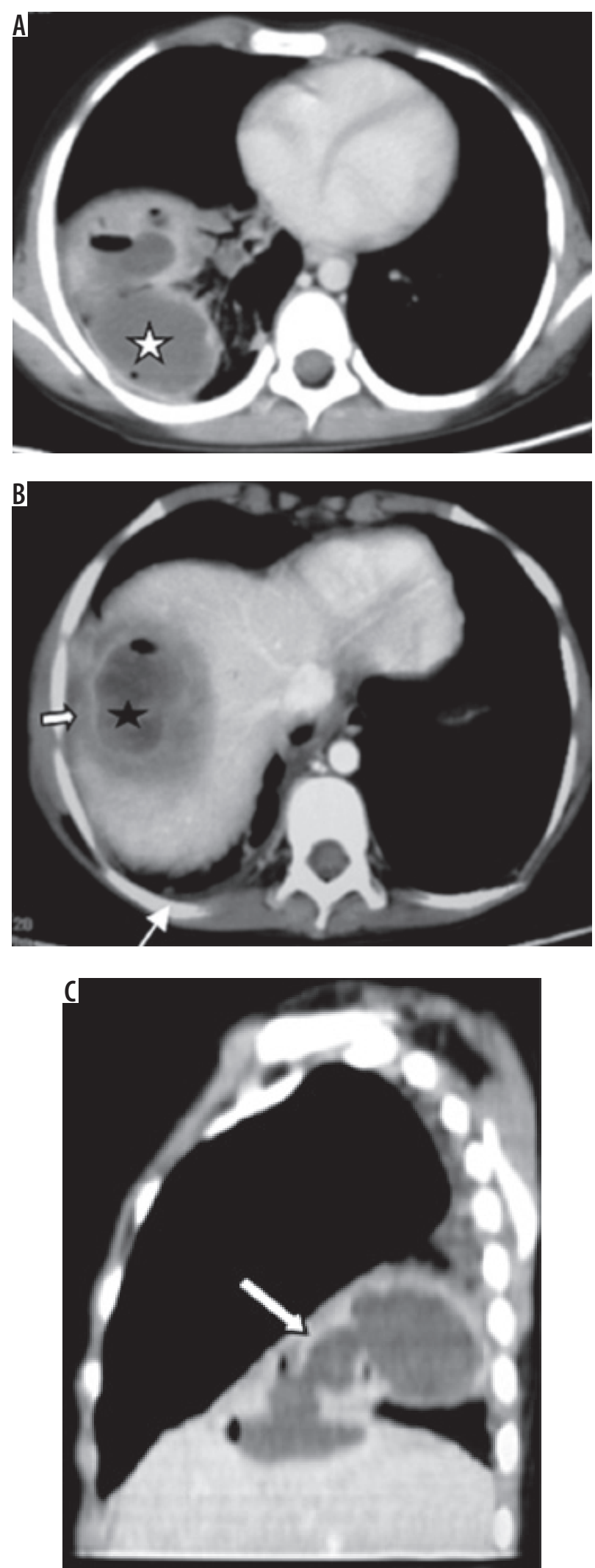

Figure 10. Patient with pain in the abdomen and fever. Diagnosed as amoebic liver abscess. A) Chest computed tomography (CT), shows presence of hypodense lesion with air fluid level in right lower lobe (white asterisk). B) Chest CT shows presence of hypodense lesion with shaggy walls in right lobe of liver with few air foci consistent with liver abscess (black asterisk). Note mild perihepatic fluid (white short block arrow) and minimal right pleural effusion (white thin arrow). C) Chest CT sagittal reformatted image, showing communication of liver abscess with lung abscess (white long block arrow) 

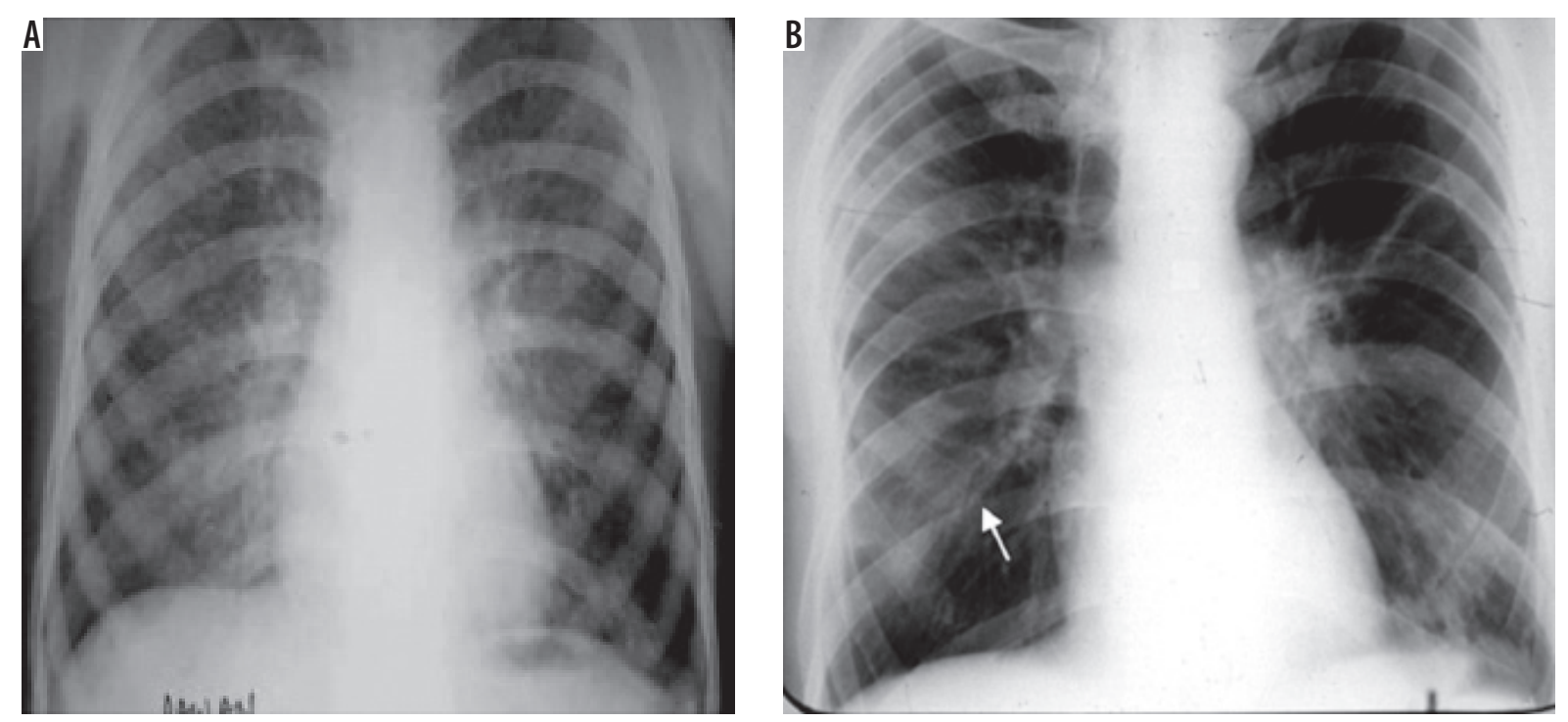

Figure 11. Löffler's syndrome. A) Chest radiograph shows presence of reticulonodular opacities in bilateral lungs. B) Chest radiograph in another patient showing mild peribronchial thickening (white arrow) and reticular opacities predominant in bilateral perihilar location and in bilateral mid and lower zones

\section{References}

1. Padley SPG, Rubens MB. Pulmonary Infections. In: Sutton D. Textbook of Radiology and Imaging. Churchill Livingstone, Edinburgh 2003; 131-160.

2. Prasad A, Agarwal K, Deepak D, Atwal SS. Pulmonary Aspergillosis: What CT can Offer Before it is too Late! J Clin Diagn Res 2016; 10: TE01-TE05.

3. Franquet T, Müller NL, Oikonomou A, Flint JD. Aspergillus infection of the airways: computed tomography and pathologic findings. J Comput Assist Tomogr 2004; 28: 10-16.

4. Henzler C, Henzler T, Buchheidt D, et al. Diagnostic Performance of Contrast Enhanced Pulmonary Computed Tomography Angiography for the Detection of Angioinvasive Pulmonary Aspergillosis in Immunocompromised Patients. Sci Rep 2017; 7: 4483.

5. $\mathrm{Hu} \mathrm{Z}$, Chen J, Wang J, et al. Radiological characteristics of pulmonary cryptococcosis in HIV-infected patients. PLoS One 2017; 12: e0173858.

6. Chang WC, Tzao C, Hsu HH, et al. Pulmonary cryptococcosis: comparison of clinical and radiographic characteristics in immunocompetent and immunocompromised patients. Chest 2006; 129: 333-340.

7. Choe YH, Moon H, Park SJ, et al. Pulmonary cryptococcosis in asymptomatic immunocompetent hosts. Scand J Infect Dis 2009; 41: 602-607.

8. Franquet T. Pulmonary Infections in Adults. In: Adam A, Dixon AK, Gillard JH, Schaefer-Prokop CM. Grainger \& Allison's Diagnostic Radiology. 6th Ed. Churchill Livingstone, Edinburgh 2014; 246266.

9. Franquet T, Giménez A, Hidalgo A. Imaging of opportunistic fungal infections in immunocompromised patient. Eur J Radiol 2004; 51: 130-138.

10. Hidalgo A, Falco V, Mauleon S, et al. Accuracy of high-resolution $\mathrm{CT}$ in distinguishing between Pneumocystis carinii pneumonia and
non-Pneumocystis carinii pneumonia in AIDS patients. Eur Radiol 2003; 13: 1179-1184.

11. Chung JH, Godwin JD, Chien JW, Pipavath SJ. Case 160: Pulmonary mucormycosis. Radiology 2010; 256: 667-670.

12. Choo JY, Park CM, Lee HJ, et al. Sequential morphological changes in follow-up CT of pulmonary mucormycosis. Diagn Interv Radiol 2014; 20: 42-46.

13. Maturu VN, Agarwal R. Reversed halo sign: a systematic review. Respir Care 2014; 59: 1440-1449.

14. Jain D, Ghosh S, Teixeira L, Mukhopadhyay S. Pathology of pulmonary tuberculosis and non-tuberculous mycobacterial lung disease: Facts, misconceptions, and practical tips for pathologists. Semin Diagn Pathol 2017; 34: 518-529.

15. Loddenkemper R, Lipman M, Zumla A. Clinical Aspects of Adult Tuberculosis. Cold Spring Harb Perspect Med 2015; 6: a017848.

16. Lyon SM, Rossman MD. Pulmonary Tuberculosis. Microbiol Spectr 2017; 5 .

17. Bhalla AS, Goyal A, Guleria R, Gupta AK. Chest tuberculosis: Radiological review and imaging recommendations. Indian J Radiol Imaging 2015; 25: 213-225.

18. Bomanji JB, Gupta N, Gulati P, Das CJ. Imaging in tuberculosis. Cold Spring Harb Perspect Med 2015; 5. pii: a017814.

19. Restrepo CS, Katre R, Mumbower A. Imaging Manifestations of Thoracic Tuberculosis. Radiol Clin North Am 2016; 54: 453-473.

20. Rozenshtein A, Hao F, Starc MT, Pearson GD. Radiographic appearance of pulmonary tuberculosis: dogma disproved. AJR Am J Roentgenol 2015; 204: 974-978.

21. Pathak V, Shepherd RW, Shojaee S. Tracheobronchial tuberculosis. J Thorac Dis 2016; 8: 3818-3825.

22. Kim JY, Jeong YJ, Kim KI, et al. Miliary tuberculosis: a comparison of CT findings in HIV-seropositive and HIV-seronegative patients. Br J Radiol 2010; 83: 206-211. 
23. Skoura E, Zumla A, Bomanji J. Imaging in tuberculosis. Int J Infect Dis 2015; 32: 87-93.

24. Reich JM. Pathogenesis of Lady Windermere syndrome. Scand J Infect Dis 2012; 44: 1-2.

25. Yuan MK, Chang CY, Tsai PH, et al. Comparative chest computed tomography findings of non-tuberculous mycobacterial lung diseases and pulmonary tuberculosis in patients with acid fast bacilli smear-positive sputum. BMC Pulm Med 2014; 14: 65.

26. Kim C, Park SH, Oh SY, et al. Comparison of chest CT findings in nontuberculous mycobacterial diseases vs. Mycobacterium tuberculosis lung disease in HIV-negative patients with cavities. PLoS One 2017; 12: e0174240.

27. Kwak N, Lee CH, Lee HJ, et al. Non-tuberculous mycobacterial lung disease: diagnosis based on computed tomography of the chest. Eur Radiol 2016; 26: 4449-4456.
28. Polat P, Kantarci M, Alper F, et al. Hydatid disease from head to toe. RadioGraphics 2003; 23: 475-494.

29. Martinez S, Restrepo CS, Carrillo JA, et al. Thoracic manifestations of tropical parasitic infections: a pictorial review. Radiographics 2005; 25: 135-155.

30. Garg MK, Sharma M, Gulati A, et al. Imaging in pulmonary hydatid cysts. World J Radiol 2016; 8: 581-587.

31. Shamsuzzaman SM, Hashiguchi Y. Thoracic amebiasis. Clin Chest Med 2002; 23: 479-492.

32. Akuthota P, Weller PF. Eosinophilic pneumonias. Clin Microbiol Rev 2012; 25: 649-660.

33. Franquet T. Imaging of pneumonia: trends and algorithms. Eur Respir J 2001; 18: 196-208. 